\title{
Fibrin degradation products in the cerebrospinal fluid of patients with pneumococcal meningitis
}

\author{
P. G. CLELA N D, J.T. MACFAR LA N E, D. R. B A I R D, \\ A N D B. M. GR E E N W O O D
}

From the Department of Medicine, Ahmadu Bello University, Zaria, Nigeria

S U M MARY Raised levels of fibrin degradation products were found in the cerebrospinal fluid of nearly all of 35 patients with pneumococcal meningitis. The mean level was higher in patients who died subsequently than in those who survived. Cerebrospinal fluid from patients with pneumococcal meningitis showed increased fibrinolytic activity as assessed by clot lysis, suggesting local production of fibrin degradation products within the subarachnoid space.

The mortality from pneumococcal meningitis remains high, especially in the tropics (Baird et al., 1976). In survivors, the illness is often protracted, and many patients are left with a permanent neurological deficit. The reasons for this high mortality and morbidity are unclear but vascular damage, occurring early in the course of the infection, may be an important factor. Postmortem studies (Cairns and Russell, 1946; Dickson and Yassin, 1969) have shown a severe vasculitis of meningeal and cerebral blood vessels with vessel wall oedema, necrosis, fibrin deposition and infiltration with inflammatory cells. Changes of such severity are rarely seen in other types of pyogenic meningitis.

We have tried to assess the possible importance of vasculitis in the pathogenesis of this condition by comparing levels of fibrin degradation products (FDP) in the cerebrospinal fluid (CSF) of patients with pneumococcal or meningococcal meningitis. The fibrinolytic properties of CSF obtained from patients with these two forms of meningitis have also been compared.

\section{Patients and methods}

PATIENTS

Thirty-five adult patients with pneumococcal meningitis and 49 adult patients with meningococcal meningitis were studied. Diagnosis was

Address for reprint requests: Dr P. G. Cleland, Department of Neurology, Middlesbrough General Hospital, Middlesbrough, Cleveland TS5 5AZ.

Accepted 17 March 1979 established by the detection of polysaccharide antigen in the CSF by counter-current immunoelectrophoresis or by latex test (Whittle et al., 1974) or by a positive CSF culture. All patients received large doses of penicillin and any other supportive treatment necessary. Seven patients with pneumococcal meningitis received intravenous heparin for five days during a drug trial (Macfarlane et al., 1977).

\section{SAMPLES}

On admission $2 \mathrm{ml}$ of lumbar CSF were collected into a tube containing 20 IU of thrombin and $4 \mathrm{mg}$ of epsilon aminocaproic acid. After incubation for one hour at $37^{\circ} \mathrm{C}$ the mixture was centrifuged, and the supernatant removed and stored at $-20^{\circ} \mathrm{C}$ until tested for FDP. Fifteen patients with pneumococcal meningitis were investigated more fully during the course of a bacteriological study of patients with this condition. Serial CSF samples were collected from these patients one, two, three, and five days after the start of antibiotic treatment and again two days after the completion of therapy. Aliquots of these samples were used for FDP assay. Five normal CSF samples were obtained from patients whose initial clinical picture suggested meningitis but in whom a diagnosis of meningitis was subsequently excluded.

Supernatant samples of sterile CSF obtained from 16 patients with pneumococcal meningitis and from 17 patients with meningococcal meningitis were deep frozen immediately after collection of the sample and stored for up to six months at $-30^{\circ} \mathrm{C}$ until tested for fibrinolytic activity. 
LABORATORY METHODS

Serum and CSF fibrin degradation products were measured by a haemagglutination inhibition assay (Burroughs Wellcome). The CSF fibrinogen content was determined by radial immunodiffusion using a monospecific antifibrinogen antiserum (Hyland Laboratories). The results are expressed as a percentage of a pooled normal Nigerian plasma standard. Cerebrospinal fluid protein levels were measured by the method of Lowry (1951).

Plasmin activity was measured by the fibrin plate technique (Lassen, 1952), and CSF fibrinolytic activity was estimated by a modification of the method of Fearnley et al. (1957). A $0.2 \mathrm{ml}$ volume of normal blood was added to a series of tubes

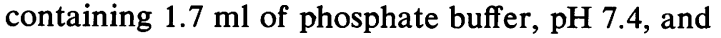
$0.1 \mathrm{ml}$ (10 IU) of thrombin. After incubation for one hour at $37^{\circ} \mathrm{C}, 0.3 \mathrm{ml}$ of buffer solution was removed carefully and replaced by $0.3 \mathrm{ml}$ of CSF. Control tubes received $0.3 \mathrm{ml}$ of buffer solution. The tubes were then incubated at $37^{\circ} \mathrm{C}$ in a water bath and the clots observed two, four, and six hours later. Clot lysis was graded as none, partial, or complete.

\section{Results}

FDP LEVELS IN CSF ON ADMISSION

Initial FDP levels in the CSF of patients with pneumococcal meningitis and meningococcal meningitis are shown in Fig. 1. The mean level found in patients with pneumococcal meningitis $(27.1 \mu \mathrm{g} / \mathrm{ml}$, SD 36.0$)$ was significantly higher than the mean level found in patients with group $\mathrm{A}$ meningococcal meningitis $(2.7 \mu \mathrm{g} / \mathrm{ml}$, SD 2.5$)$ or group C meningococcal meningitis $(2.4 \mu \mathrm{g} / \mathrm{ml}$, SD 2.8) $(\mathrm{P}<0.001$ for each comparison).

The mean initial FDP level in CSF of patients with pneumococcal meningitis who died subsequently $(43.1 \mu \mathrm{g} / \mathrm{ml}$, SD 42.3) was significantly higher than the mean initial FDP level in CSF of those who survived $(8.1 \mu \mathrm{g} / \mathrm{ml}$, SD 11.8$)(\mathrm{P}<0.001)$ (Fig. 2).

SERIAL FDP AND FIBRINOGEN LEVELS IN CSF

Figure 3 shows the sequential CSF and serum FDP levels observed in 15 patients with pneumococcal meningitis during treatment. Five patients died during the study period. During the early phase of the illness, FDP levels in CSF were consistently higher than in serum. Overall some correlation was found between CSF and serum values $(r=0.56 ; \mathrm{P}<0.01)$.

Serial FDP and fibrinogen levels in CSF are shown in Fig. 4. A highly significant correlation

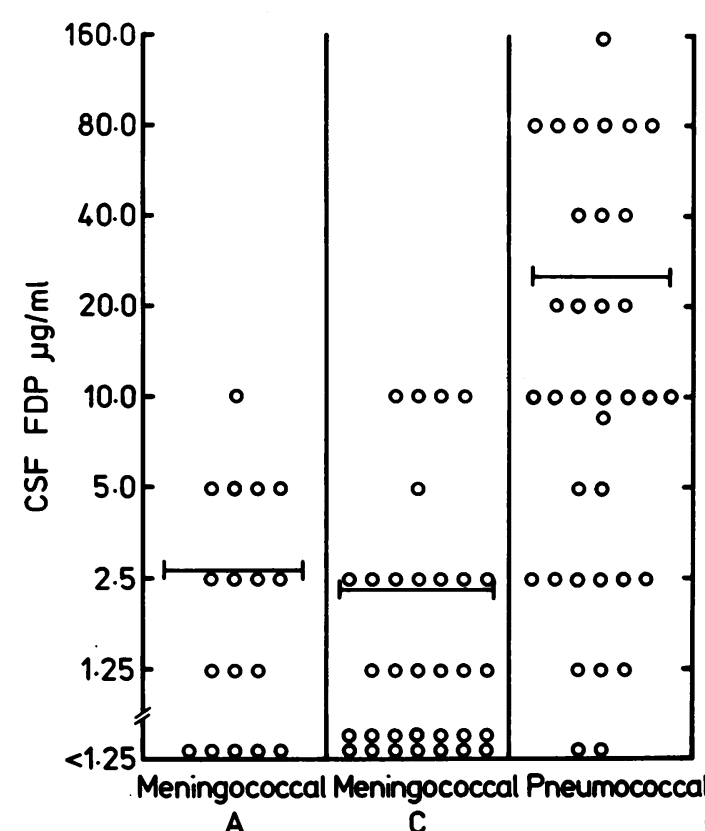

Fig. 1 Initial CSF FDP levels in patients with pneumococcal or meningococcal meningitis.

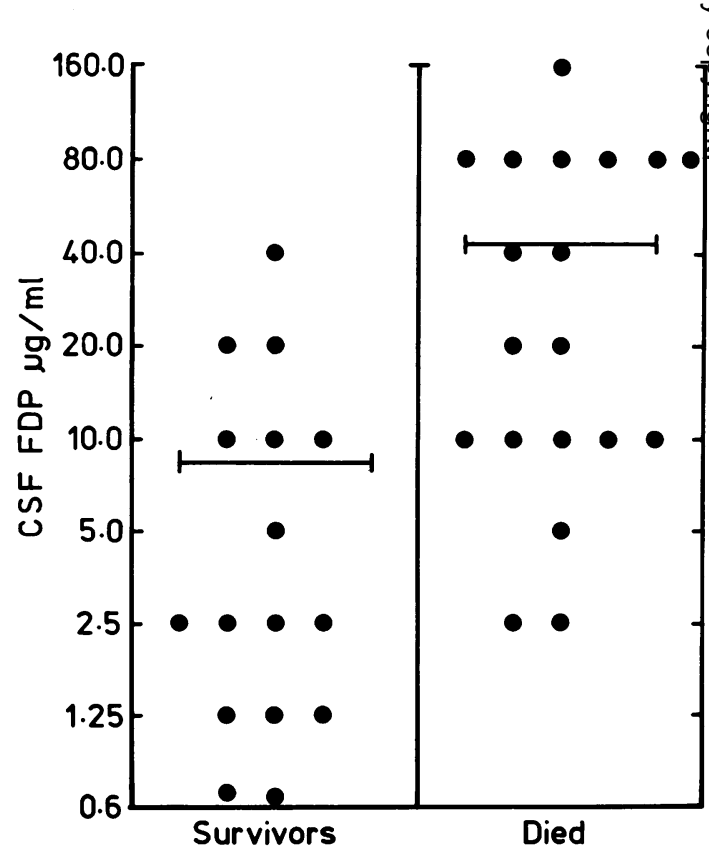

Fig. 2 Initial CSF FDP levels in 19 patients with pneumococcal meningitis who died subsequently and in 15 patients who survived. 


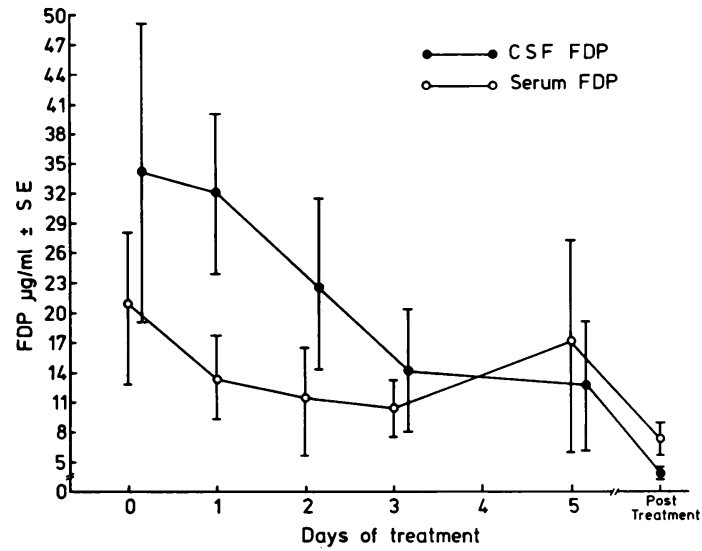

Fig. 3 Serial CSF and serum FDP levels in 15 patients with pneumococcal meningitis during the course of treatment.

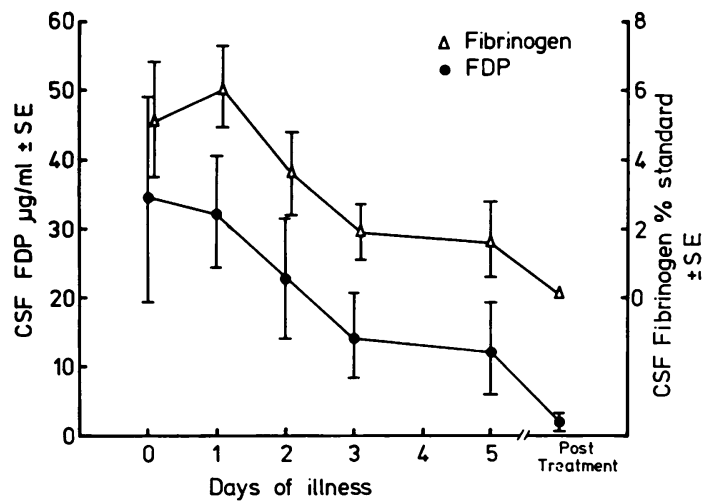

Fig. 4 Serial CSF FDP and fibrinogen levels in 15 patients with pneumococcal meningitis during treatment.

was found between the two $(r=0.86 ; \mathrm{P}<0.001)$. Although the antifibrinogen antiserum cross reacts with FDP this does not affect the correlation for the levels of fibrinogen were about one hundred times greater than FDP.

No correlation was found between CSF levels of FDP and protein.

\section{FIBRINOLYTIC ACTIVITY OF CSF}

Cerebrospinal fluid samples from patients with pneumococcal meningitis or meningococcal meningitis were tested for fibrinolytic activity, and the results of clot lysis tests are shown in the Table. Samples from patients with pneumococcal meningitis produced clot lysis more rapidly than samples from patients with meningococcal
Table Clot lysis by cerebrospinal fluid from patients with pyogenic meningitis and from control subjects

\begin{tabular}{lccc}
\hline Patients & \multicolumn{3}{l}{ Incubation time (hr) } \\
\cline { 2 - 4 } & Two & Four & Six \\
\hline Pneumococcal (16) & 16 & 6 & 0 \\
$\quad$ None & 0 & 4 & 4 \\
Partial & 0 & 6 & 12 \\
$\quad$ Complete & & & \\
Meningococcal (17) & 17 & 15 & 2 \\
$\quad$ None & 0 & 1 & 6 \\
$\quad$ Partial & 0 & 1 & 9 \\
$\quad$ Complete & & & \\
Control subjects (5) & 5 & 5 & 5 \\
$\quad$ None & & & \\
\hline
\end{tabular}

meningitis. The difference between the two groups after four hours of incubation is statistically significant $(P=0.01)$. Control CSF samples showed no clot lysis at six hours, but in all cases lysis was complete after $24 \mathrm{hr}$ of incubation. Using the fibrin plate technique plasmin activity was detected in one of 16 samples from patients with pneumococcal meningitis but in none of $17 \mathrm{CSF}$ samples obtained from patients with meningococcal meningitis.

\section{Discussion}

This study has shown that the level of FDP in the CSF is higher in patients with pneumococcal meningitis than in patients with meningococcal meningitis. The CSF level reflects the severity of the disease, for high values were found in patients who died subsequently.

The source of the FDP found in the CSF of patients with meningitis is not known. They are not found in normal CSF but have been detected in the CSF of patients with a wide variety of noninfective neurological conditions (Brueton et al., 1976; Anderson et al., 1978). The presence of FDP, and other low molecular weight proteins, in the CSF of these patients is thought to follow their leakage from the plasma across a damaged blood-brain barrier (Anderson et al., 1978). However, we think that this is unlikely to be the whole explanation of the high level of FDP found in the CSF of patients with pneumococcal meningitis for in these patients CSF levels are often considerably higher than serum levels. We think that it is more likely that the FDP found in the CSF of patients with pneumococcal meningitis are formed in addition locally as a result of fibrinolysis of fibrinogen and fibrin within the subarachnoid space.

Cerebrospinal fluid from patients with pneumococcal meningitis had increased fibrinolytic activity 
as assessed by clot lysis. This lysis was probably due to the presence of plasminogen activator rather than plasmin as there was no lysis of fibrin plates. Plasminogen activator is not usually found in the CSF (Tovi, 1972) but it is present in the meninges and choroid plexus. Pneumococci do not produce a plasminogen activator in tissue culture but it is possible that they can induce the release of activator from the meninges into the CSF. That the FDP are produced by lysis of fibrinogen and fibrin is supported by our demonstration of a close relationship between CSF levels of FDP and fibrinogen.

The results of this study give some support to the view that local vascular damage and extravascular fibrin deposition contribute to the poor outcome of patients with pneumococcal meningitis. An attempt to prevent the development of these changes by administration of heparin was unsuccessful (Macfarlane et al., 1977) but a controlled trial of a fibrinolytic agent might be worthwhile.

We thank Professor A. A. Fleming and Dr H. C. Whittle for their helpful comments, Professor E. H. O. Parry for his encouragement, and our clinical colleagues who allowed us to study patients under their care. BMG is supported by the United Kingdom Medical Research Council.

\section{References}

Anderson, M., Matthews, K. B., and Stuart, J. (1978). Coagulation and fibrinolytic activity of cerebrospinal fluid. Journal of Clinical Pathology, 31, 488-492.
Baird, D. R., Whittle, H. C., and Greenwood, B. M. (1976). Mortality from pneumococcal meningitis. Lancet, 2, 1344-1346.

Brueton, M. J., Breeze, G. R., and Stuart, J. (1976). Fibrin-fibrinogen degradation products in cerebrospinal fluid. Journal of Clinical Pathology, 29, 341344.

Cairns, H., and Russell, D. S. (1946). Cerebral arteritis and phlebitis in pneumococcal meningitis Journal of Pathology and Bacteriology, 58, 649-665.

Dickson, L. G., and Yassin, M. W. (1969). Meningeal vascular thrombosis in pneumococcal meningitis. Journal of the Egyptian Public Health Association, 44, 349-355.

Fearnley, G. R., Balmforth, G., and Fearnley, E. (1957). Evidence of a diurnal fibrinolytic rhythm with a simple method of measuring natural fibrinolysis. Clinical Science, 16, 645-650.

Lassen, M. (1952). Heat denaturation of plasminogen in the fibrin plate method. Acta Physiologica Scandinavica, $27,371$.

Lowry, O. M., Rosebrough, N. J., Farr, A. L., and Randall, R. J. (1951). Protein measurement with the folin phenol reagent. Journal of Biological Chemistry, 193, 265-275.

Macfarlane, J. T., Cleland, P. G., Attai, E., and Greenwood, B. M. (1977). Failure of heparin tob $\frac{D}{\omega}$ alter the outcome of pneumococcal meningitis $\mathbb{D}$ British Medical Journal, 2, 1522.

Tovi, D. (1972). Studies of fibrinolysis in the centrap nervous system with special reference to intracrania $\mathcal{P}$ haemorrhage and to the effect of antifibrinolytion drugs. Umea University Medical Dissertations, 8 $1-50$.

Whittle, H. C., Tugwell, P., Egler, L. J.., and Green wood, B. M. (1974). Rapid bacteriological diagnosis of pyogenic meningitis by latex agglutination. Lancet, 2, 619-621. 